\title{
A Coupling Method for Identifying Arc Faults Based on Short-Observation-Window SVDR
}

\author{
Run Jiang, Guanghai Bao, Qiteng Hong and Campbell Booth
}

\begin{abstract}
This paper presents a new method for effective detection of AC series arc fault (SAF) and extraction of SAF characteristics in residential buildings, which addresses the challenges with conventional current detection methods in discriminating arcing and non-arcing current due to their similarity. Different from the traditional method, in the proposed method, the differential magnetic flux is coupled to obtain high-frequency signals by putting the live line and the neutral line through the current transformer, which can effectively solve the problem of SAF features disappearing in the trunk-line current. However, similar to the traditional method, the effectiveness of the proposed coupling method could also be compromised when being used in cases with dimmer load and load starting process. This is found to be caused by the presence of high-amplitude pulse phenomenon in the non-arcing signals in these scenarios, which are incorrectly detected as arcing signals in other loads. To address this issue, a short-observation-window singular value decomposition and reconstruction algorithm (SOW-SVDR) is used to enhance the capability to identify SAFs by the coupling method. The proposed method has been implemented and validated according to UL1699 standard with different types of loads connected to the system and also tested under their starting processes. The experimental results show that the proposed approach is more effective in detecting arc faults compared with existing methods.
\end{abstract}

Index Terms-Series arc fault, coupling signals, shortobservation-window singular value decomposition and reconstruction (SOW-SVDR), UL1699.

\section{INTRODUCTION}

A $\mathrm{C}$ arc faults (AFs) often appear in residential buildings due to non-standard operation of appliances, aged cable insulation, etc. During AFs, the temperature can reach a very high level, i.e. in the range of thousands of degrees Celsius, which can easily lead to fire hazards. According to the latest electrical fire reports from China Fire Yearbook and U.S. Fire Administration, the proportion of casualties and property losses caused by electrical fires still keeps very high. Therefore, it is critical to effectively detect arc faults in a timely manner in order to minimize the risk of fire accidents. AFs are divided into series arc faults (SAFs) and parallel arc faults (PAFs). For PAFs, the current signals are relatively obvious and can be easily discriminated from normal (non-arcing) ones [1].

Run Jiang and Guanghai Bao are with College of Electrical Engineering, Fuzhou University, Fuzhou 350108, China (Corresponding author: Guanghai Bao; e-mail: 19428733@qq.com).

Qiteng Hong and Campbell Booth are with Department of Electronic \& Electrical Engineering, University of Strathclyde, Glasgow, G1 1XW, UK
However, correct detection and identification of SAFs remain to be challenging [2]. The reasons can be listed as follows. Firstly, SAF current waveforms can be significantly different when different loads and their combination are connected in the system and the SAF characteristics can be very similar to the current waveforms in some normal-operating appliances, such as resistive loads and dimmer loads. Secondly, SAF characteristics are susceptible to the parallel circuit and often disappear especially when the fault branch is in parallel with the resistive load. Moreover, SAF current waveforms are impossible to stay invariable even with the same load, which makes SAF identification extremely challenging. Therefore, most researchers focus on not only current signals, but also other types of signals for SAF detection. In general, the SAF detection methods can be divided into two main categories, i.e. the physical signal detection methods and the electrical signal detection methods. The former methods use signals including voice, light, heat, electromagnetic radiation and ultraviolet for SAF detection [3]. The latter methods are mainly based on current and voltage signals [4-5].

Since AFs can generate light and voice when they occur, reference [6] has used the two signals to identify AFs, which can achieve a detection time within $4 \mathrm{~ms}$ in industries. Reference [7] has made use of the length of ultraviolet and acoustic emission to detect arcs in the low-voltage switchboards. However, these methods have certain shortcomings as their performance can be severely affected by other normal signals and the place where arcs occur. To solve the problems, the detection on electromagnetic radiation is proposed to acquire useful signals produced by AFs [8-10]. Compared with the above-mentioned methods, the current signal-based AF detection method is considered to be more promising, because many references prove that there is more abundant information in current which can directly reflect whether there is an arc. To extract unique arcing current characteristics, researchers have introduced a wide range of algorithms, which can be classified into three types: time-domain analysis, frequency-domain analysis and time-frequency-domain analysis. In the time domain, arcing current has the shoulders, which mean current value is nearly zero when it is around normal zero crossing [11]. However, this feature is not suitable to be used for AF detection, because there is the same phenomenon in the current when the dimmer load is working under the normal state. Some papers extract high-frequency pulses by derivative of current, but fail to work in the dimmer loads or the corresponding combination loads 
[12-13]. Although RMS of arcing current is also a noticeable feature and is lower than that of normal current, it can easily lead to mal-detection of AFs when there are normal voltage fluctuations. Due to the limit of the time-domain signals, some papers turn to the frequency-domain analysis, because the frequency range of AFs is wide, which can reach almost $\mathrm{MHz}$ [14]. Fast Fourier Transformation (FFT) is commonly used in frequency-domain analysis [15]. However, AF detection requires a relatively short observation window for fast identifying arcs. In FFT, a short observation window will result in a low frequency resolution and can cause spectrum leakage. Therefore, frequency refinement technology, known as zero padding, Zoom-FFT (ZFFT) and Chirp-Z Transformation (CZT), is often used to replace FFT. Actually, only time-domain analysis or frequency-domain analysis is not sufficient, and most papers prefer to combine both of them. Reference [16] has analyzed low-frequency harmonic components based on CZT, from $0 \mathrm{~Hz}$ to $500 \mathrm{~Hz}$, and difference of current signals. Reference [17] proposes to detect SAFs in some loads by current amplitude spectrum and norm-based sparse representation. Final tests show L3/4 norm is the best parameter and its detection accuracy can reach a high level. In addition to the aforementioned transformation algorithms, Wavelet Transformation (WT) and Stockwell Transformation (ST) are also used to detect SAFs, as they can retain the time-domain information [18-21]. In WT, wavelet basis selection is critical for the detection performance. Reference [22] proves that Db13 is a better choice for SAF detection. Reference [23] has analyzed arcing waveform distortion by Db4-based WT and an improved arc model. In addition, it has also analyzed harmonic energy by FFT. Recently, artificial intelligence-based (AI) algorithms become increasingly popular. In machine learning (ML), softmax regression, neural network (NN) with softmax activation function, is often used for binary classification, which means that 1 and 0 stand for arcs and no arcs, respectively. Reference [24] has decomposed current signals by wavelet package. Then, the fault features extracted from reconstructed current signals are used as the input of support vector machine. Reference [25] has proposed to recognize current category before the customized features are imported to the full-connected NN. Due to the complexity of arcing signals, the NN could be highly complicated, to some extent, to enhance detection accuracy by increasing the number of input characteristics, units and layers, which could require significant computation capability and storage capacity to run the algorithms [26-29]. In order to extract more features, NN with convolutional layers $(\mathrm{CNN})$ is proposed, e.g. VGG, AlexNet, etc. [30], and these methods could make the AF detection task more time-consuming. It should be noted that, in these methods, hyperparameters need to be constantly adjusted so as to find out relatively appropriate values for identifying SAFs and solve over-fitting and under-fitting issues, which could be significantly time-consuming. Furthermore, it is also likely that the proposed NN or CNN might not work not well after a certainly period of time because of variable circuits. As a result, the $\mathrm{NN}$ or $\mathrm{CNN}$ has to be re-adjusted, along with a series of actions, e.g. data augmentation [31].
To solve the problem that SAF characteristics change with loads, reference [32] has preliminarily proposed a coupling method to pass the live line and the neutral one through the current transformer to obtain the high-frequency oscillating pulses of SAFs. The currents of the two line are with same magnitude but opposite directions. In reality, the positions of the two cables are asymmetrical, so there is low-amplitude differential magnetic flux. When there are no arcs, high-frequency components of current are few, and the secondary output is nearly zero. On the contrary, the output shows many pulses. However, some vital problems, which cause a severe limitation on the general applicability of the coupling method, are still not addressed:

(1) The coupling method is not effective when there is presence of the dimmer loads and it could mal-detect AFs during normal operation, because the output waveform contains high-amplitude pulses, which can be confused with that in the fault condition.

(2) Similar to the traditional current detection method, the coupling method also experiences mal-detection of AFs during in the starting process of loads. Many papers have to increase the number of detection cycles to avoid misjudgment from the starting process.

Therefore, in this paper, the aforementioned limitations have been fully addressed, and the proposed method offers a promising solution for effective SAF detection while being capable of avoiding mal-detection in systems with dimmer loads and during load staring processes. The main contributions and are summarized as follows:

In Section II, the main contribution of the coupling method is clearly demonstrated by introducing the flaws of the current detection method, which is totally different from reference [32]. Then, although the coupling method can realize classification effect to distinguish between arcing signals and non-arcing ones in most loads, it fails to work in the dimmer loads, where the normal signals are similar to the fault ones. Therefore, in Section III, the coupling method based on a short-observation -window singular value decomposition and reconstruction algorithm is proposed to achieve correct classification for arc fault detection. The algorithm can filter out the non-arcing pulses and retain the arcing pulses to promise the detection reliability. Meanwhile, SOW-SVDR can weaken the influence from the appliance staring process to a large extent and decrease the additional detection cycles used for it. Finally, the experimental results show that the proposed detection strategy can effectively identify arc faults in Section IV.

\section{ARC FAULT DETECTION METHODS}

\section{A. Limitation of Traditional Current Detection Method}

When SAFs occur, there are some corresponding fault characteristics in the load current. In order to extract them, most papers analyze the difference between non-arcing current and arcing current in the time domain and the frequency domain. However, the authors have found that the arcing characteristics, in the detected current, tend to disappear when one branch where SAFs occur is in parallel with another normal one. To 
illustrate the phenomenon, an incandescent lamp (200W) is chosen for the fault branch load, and a resistance $(22 \Omega)$ is chosen for the normal branch load as shown in Fig. 1(a). The waveforms of the trunk current under no arcs and arcs are shown in Fig. 1 (b) and (c) respectively. It can be seen from Fig. 1 that there is no clear difference between the arcing waveform and the non-arcing one, as the arcing characteristics are so small that they may not be noticeable in the trunk current when the trunk current amplitude is high. Under this circumstance, whether time-domain analysis or frequency-domain analysis will fail to extract arcing features. Fig. 2 presents the methods based on the first derivative and CZT respectively, which try to obtain unique features of arcing current in Fig. 1 (c). The first derivative is an algorithm commonly used to obtain arcing pulses in many papers [12-13]. The amplitude in Fig. 2 (a) and (b) is limited to $1 \times 10^{6}$ because of the $1 \mathrm{MHz}$ sampling rate. In $\mathrm{CZT}$, high frequency resolution (FR) is adopted to obtain detailed information of SAFs in the frequency band of [0-500] $\mathrm{Hz}$, which has been used in [16]. The observation window is $0.1 \mathrm{~s}$, and the sampling rate is $1 \mathrm{MHz}$. The refining multiple is $100000(0.1 \times 1000000)$. The interesting frequency band is [0-500] Hz. Therefore, FR is $5 \mathrm{mHz}(500 / 100000)$. However, the capability of identifying SAFs are not satisfactory with either the time domain or the frequency domain. In Fig. 2, the non-arcing signals are almost the same as the arcing ones.

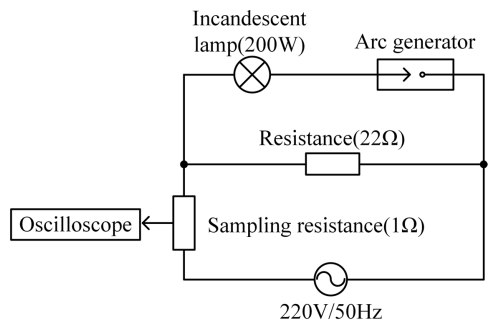

(a)

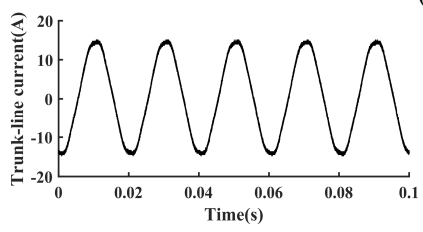

(b)

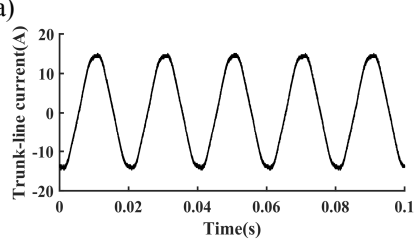

(c)
Fig. 1. Trunk current waveforms under arcs and no arcs to show the limitation in the traditional method. (a) Schematic of the experimental platform. (b) Non-arcing waveform. (c) Arcing waveform. (a)

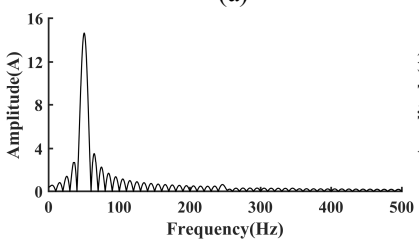

(c)

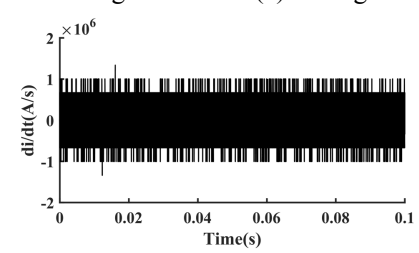

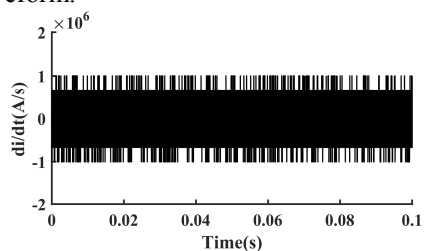

(b)

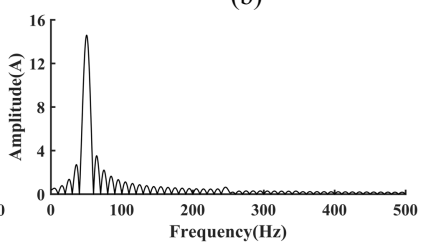

(d)

Fig. 2. Time domain analysis (di/dt) and frequency domain analysis (CZT) to show the limitation in the traditional method. (a) Waveform of the first

derivative of non-arcing current. (b) Waveform of the first derivative of arcing current. (c) Spectrum of non-arcing current. (d) Spectrum of arcing current.

\section{B. Advantage of the Proposed Coupling Method}

In order to solve the problem mentioned above, a coupling method is proposed to extract the small fault characteristics. In Fig. 3, the live line and the neutral line are passed through the current transformer. Their current amplitudes are equal, but with opposite directions. It is noted that the transformer aperture just accommodates two wires.

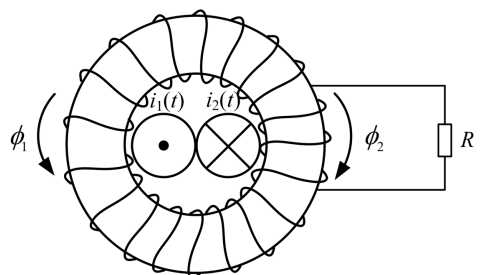

Fig. 3. Schematic of the coupling method.

The two detected currents can be expressed by Fourier expansion:

$$
\begin{aligned}
& i_{1}(t)=-i_{2}(t)=\frac{a_{0}}{2}+\sum_{m=1}^{\infty}\left[a_{m} \cos (m \omega t)+b_{m} \sin (m \omega t)\right] \\
& =\frac{a_{0}}{2}+\sum_{m=1}^{\infty} c_{m} \sin \left(m \omega t+\phi_{m}\right)=\sum_{m=0}^{\infty} c_{m} \sin \left(m \omega t+\phi_{m}\right)
\end{aligned}
$$

Where $m$ is the harmonic order, and $\omega$ is the angular frequency of the fundamental wave. The parameter $a_{0}$ is the direct current component. The parameters, $a_{0}, a_{m}, b_{m}, c_{m}$, and $\phi_{m}$, are defined as follows:

$$
\begin{aligned}
& a_{m}=\frac{1}{\pi} \int_{-\pi}^{\pi} i_{1}(t) \cos (m \omega t) d(\omega t) \\
& b_{m}=\frac{1}{\pi} \int_{-\pi}^{\pi} i_{1}(t) \sin (m \omega t) d(\omega t) \\
& C_{m}=\sqrt{a_{m}^{2}+b_{m}^{2}}, m=1, \ldots, \infty . \\
& \phi_{m}=\arcsin \left(\frac{a_{m}}{\sqrt{a_{m}^{2}+b_{m}^{2}}}\right) \\
& C_{0} \sin \phi_{0}=\frac{a_{0}}{2}, \quad a_{0}=\frac{1}{\pi} \int_{-\pi}^{\pi} i_{1}(t) d(\omega t)
\end{aligned}
$$

Therefore, their corresponding magnetic flux can be approximately expressed below:

$$
\begin{aligned}
& \phi_{1}=\sum_{m=0}^{\infty} k_{m}^{+} C_{m} \sin \left(m \omega t+\phi_{m}\right) \\
& \phi_{2}=\sum_{m=0}^{\infty} k_{m}^{-} C_{m} \sin \left(m \omega t+\phi_{m}\right)
\end{aligned}
$$

Where $k_{m}^{+}$and $k_{m}^{-}$are linear parameters. Then, according to the law of electromagnetic induction, the output voltage $u(t)$ can be obtained:

$$
u(t)=\frac{d\left(\sum_{m=0}^{\infty}\left(k_{m}^{+}-k_{m}^{-}\right) C_{m} \sin \left(m \omega t+\phi_{m}\right)\right)}{d t}
$$


In reality, $k_{m}^{+}$is not equal to $k_{m}^{-}$due to the asymmetrical positions of the two cables, and their difference value is expected to be very small. Assuming the value can be replaced by a small-value constant $\chi$ which is not related to the harmonic order:

$$
u(t) \approx \chi \frac{d i_{1}(t)}{d t}
$$

As it can be seen from the above mentioned equation, the coupling output voltage is a small-value constant multiplies the derivative result of the trunk current. It means that the coupling method can extract high-slope arcing pulses (high-frequency components) and filter out low-frequency current, which is verified in Fig. 4.

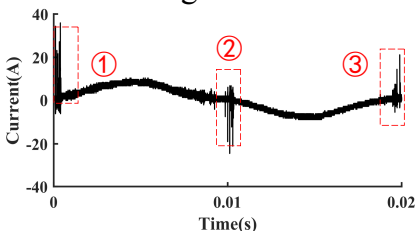

(a)

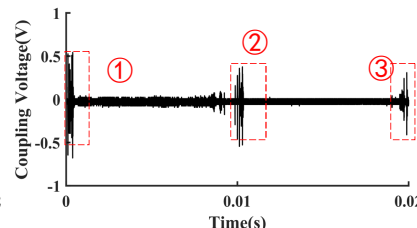

(b)
Fig. 4. Verification of the equation (5). (a) Arcing current waveform of the vacuum cleaner. (b) Corresponding coupling voltage waveform.

When the detected current waveform is smooth with only low-frequency components, the output voltage is nearly zero. On the contrary, the output voltage is not zero when there are high-frequency components in the detected current. This principle provides an ideal indicator for identifying SAFs in most loads, because there are abundant high-frequency pulses in arcing current [33]. In Fig. 1 (a), the resistance current is $i_{\text {resi }}$ and its maximum angular frequency is $\omega_{1}$. The incandescent lamp current is $i_{\text {inca }}$ and its maximum angular frequency is $\omega_{2}$. When SAFs occur, $\omega_{2}$ is much larger than $\omega_{1}$, and $u(t)$ can be written as:

$$
u(t) \approx \chi \frac{d\left(i_{\text {resi }}+i_{\text {inca }}\right)}{d t} \approx \chi \frac{d i_{\text {inca }}}{d t}
$$

In the equation (6), the derivative result of $i_{\text {resi }}$ is a small-amplitude signal, which can be neglected because of $\chi$ and its low-frequency components. Therefore, that is the reason why the coupling method can extract the arcing features which are not noticeable and difficult to detect in the trunk current. Fig. 5 (b) shows that the proposed coupling method can solve the problems in the traditional current detection method of Fig. 5 (a). By the coupling method, there is clear difference between arcs and no arcs in Fig. 5 (b). (a)

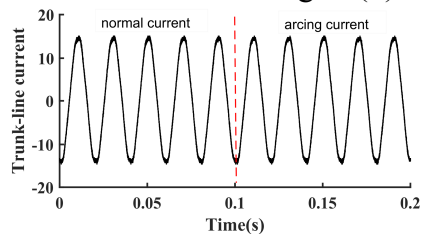

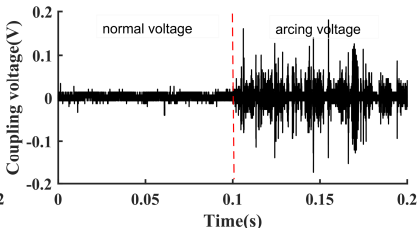

(b)
Fig. 5. Comparison between the traditional current detection method and the proposed coupling method. (a) Trunk-line current waveform before and after arc occurrence. (b) Corresponding coupling voltage waveform.
TABLE I

COMPARISON BetweEn TRAditional Method AND COUPLing METHOD

\begin{tabular}{cc}
\hline \hline Method & $\begin{array}{c}\text { Ability to extract small- } \\
\text { amplitude arcing features: } \\
\left(E_{\text {arcing }}-E_{\text {non-arcing }}\right) / E_{\text {non-arcing }}\end{array}$ \\
\hline $\begin{array}{c}\text { Current detection with time-domain } \\
\text { analysis (di/di algorithm) }\end{array}$ & $<0.01$ (Weak) \\
$\begin{array}{c}\text { Current detection with } \\
\text { frequency-domain analysis (FFT } \\
\text { algorithm: FR=1Hz) }\end{array}$ & $<0.01$ (Weak) \\
Current detection with \\
frequency-domain analysis (CZT \\
algorithm: FR=5mHz) \\
$\begin{array}{c}\text { Current detection with } \\
\text { time-frequency-domain analysis } \\
\text { (Db4/Db13-based WT algorithm) }\end{array}$ \\
Coupling voltage detection \\
\hline \hline
\end{tabular}

Finally, in the aspect of extracting small-amplitude arcing features, the comparison between the traditional method and the proposed one is shown in Table I. In the traditional method, the authors have tried numerous algorithms proposed in existing publications, including FFT, WT and CZT, in order to solve the problem that arcing characteristics disappear in the trunk-line current, but none of them provides satisfactory performance. In Table I, a quantitative parameter is used to confirm the ability to extract small-amplitude arcing features. Since the arcing waveform contains many pulses, the difference value of the variance operation between the non-arcing waveform and the arcing one should be very large. The bigger value it shows, the more arcing features we can extract. As a comparison, the coupling method proposed in this paper shows much stronger capability in SAF detection, which successfully addresses the limitations in the aforementioned algorithms and provides a promising solution for effective SAFs detection. The comparison in Table I can be also verified in Fig. 1,2 and 5.

\section{Coupling Method Based on Singular Value DECOMPOSITION AND RECONSTRUCTION}

Fig. 6 shows the coupling waveforms under arcs and no arcs, respectively.

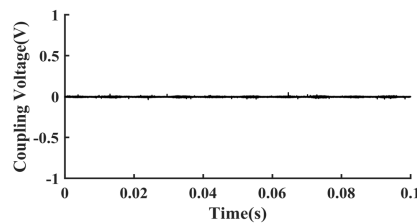

(a)

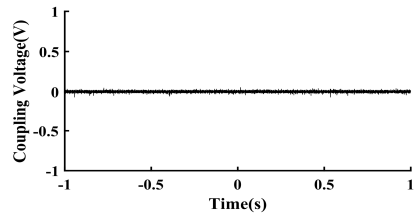

(c)

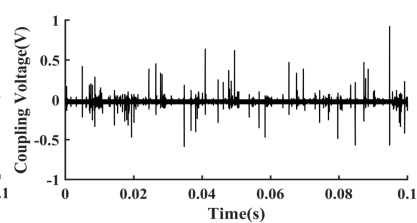

(b)

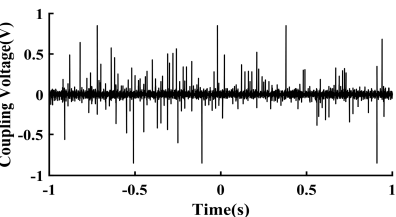

(d)
Fig. 6. Coupling voltage waveforms under arcs and no arcs, respectively. (a) Normal waveform in the computer load. (b) Fault waveform in the computer load. (c) Normal waveform in the load of fluorescent lamps. (d) Fault waveform in the load of fluorescent lamps. 
Just like Fig. 6 (a) and (c), in most loads, the non-arcing coupling waveforms are stable signals, nearly zero, because there are few high-frequency pulses in their current, which makes di/dt and $u(t)$ to be a small value. Just like Fig. 6 (b) and (d), the arcing coupling waveforms show fluctuation phenomenon and contain lots of pulses, because di/dt and $u(t)$ are very big, and the pulses in load current will be coupled by the proposed method. Thanks to the advantages of the proposed coupling method, it solves the problem (hard to obtain common features due to the different waveforms) in the traditional detection method mentioned in Section I . Under the proposed coupling method, there are clear fault characteristics when SAF occurs, and it is easy to judge whether there is an arc fault by some simple algorithms, such as finite difference, variance, etc.

However, when the dimmer load is connected and under normal operation, there are also high-amplitude pulses in the coupling waveform, and they are often incorrectly detected as arcing faults. The undesirable phenomenon could result in misjudgment if the aforementioned algorithms are used for identifying SAFs. As shown in Fig. 7 (a) and (b), the high rate of current rise which is caused by turning on the dimmer will be extracted by the coupling method. The non-arcing pulses in the dimmer load are similar to the arcing ones in the resistance and the air compressor. Furthermore, the frequency range of the non-arcing pulses is relatively wide (from $50 \mathrm{~Hz}$ to the range of $\mathrm{MHz}$ ), which makes it challenging to filter them out.

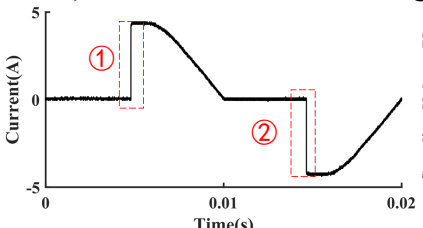

(a)

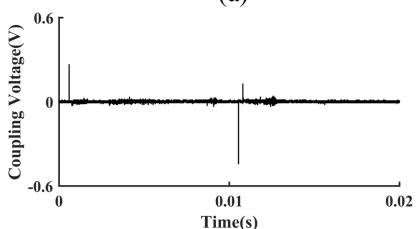

(c) (b)

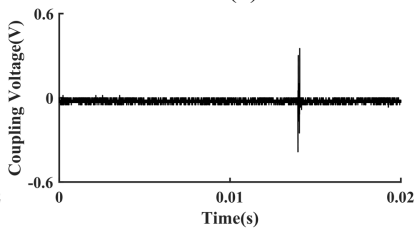

(d)

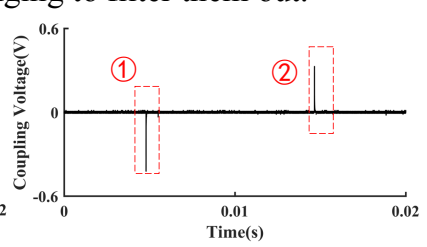

Fig. 7. Misjudgment caused by the dimmer load. (a) Normal current waveform in the dimmer load. (b) Normal coupling voltage waveform in the dimmer load. (c) Fault coupling voltage waveform in the resistance. (d) Fault coupling voltage waveform in the air compressor.

\section{A. Short-Observation-Window Singular Value Decomposition and Reconstruction (SOW-SVDR)}

Compared with eigendecomposition, SVD is a more flexible algorithm which can transform an arbitrary matrix into many components. These components stand for characteristics of the decomposed matrix. Assuming the number of samples is $k(u 1$, $\left.u 2, \ldots, u_{m}, \ldots, u_{k}\right)$ for the measured coupling signals in a short window, and a matrix $P_{n \times m}(k=n \times m)$ can be composed of these samples:

$$
P_{n \times m}=\left(\begin{array}{lll}
u_{1} & \cdots & u_{m} \\
\vdots & \ddots & \vdots \\
u_{(n-1) m+1} & \cdots & u_{k}
\end{array}\right)
$$

Then, the matrix $P_{n \times m}$ can be decomposed as follows [34]:

$$
P_{n \times m}=W_{n \times n} \cdot V_{n \times m} \cdot G_{m \times m}^{T}
$$

Where $W_{n \times n}$ and $G_{m \times m}$ are orthogonal matrixes, and $T$ represents the transpose operation. They can be obtained by the following equations [34]:

$$
\begin{gathered}
P^{T} P=G V^{T} W^{T} W V G^{T}=G V^{T} V G^{T}=G \Lambda_{1} G^{T} \\
P P^{T}=W V G^{T} G V^{T} W^{T}=W V V^{T} W^{T}=W \Lambda_{2} W^{T}
\end{gathered}
$$

Since $P^{T} P$ and $P P^{T}$ are symmetric matrixes, they can be diagonalized. Meanwhile, they share the same nonzero eigenvalues $\left(\lambda_{1} \cdots \lambda_{i}\right)$, which constitute the matrix $\Lambda_{1}$ and the matrix $\Lambda_{2}$. The columns of the matrix $G$ are composed of the eigenvectors $\left(g_{1} \cdots g_{m}\right)$ of $P^{T} P$, and the columns of the matrix $W$ are composed of the eigenvectors $\left(w_{1} \cdots w_{n}\right)$ of $P P^{T}$. Assuming that $m$ is bigger than $n$ in the matrix $P_{n \times m}$. Therefore, the equation (9) can be further expressed:

$$
\begin{aligned}
& P_{n \times m}=\left[w_{1} \cdots w_{n}\right]\left[\begin{array}{cccc}
\sigma_{1} & & 0 & \\
& \ddots & & \mid 0 \\
0 & & \sigma_{n}
\end{array}\right]\left[\begin{array}{c}
g_{1}^{T} \\
\vdots \\
g_{m}^{T}
\end{array}\right] \\
& =\left[w_{1} \cdots w_{n}\right]\left[\begin{array}{ccc}
\sigma_{1} & & 0 \\
& \ddots & 10 \\
0 & & 0
\end{array}\right]\left[\begin{array}{c}
g_{1}^{T} \\
\vdots \\
g_{m}^{T}
\end{array}\right]+\cdots+\left[w_{1} \cdots w_{n}\right]\left[\begin{array}{cccc}
0 & & 0 & \\
& \ddots & & 10 \\
0 & & \sigma_{n}
\end{array}\right]\left[\begin{array}{c}
g_{1}^{T} \\
\vdots \\
g_{m}^{T}
\end{array}\right] \\
& \sigma_{i}=\sqrt{\lambda_{i}}, i=1 \cdots n
\end{aligned}
$$

Where $\sigma_{i}$ is called the singular value. In the matrix $V$, the singular values are put in the descending order, $\sigma_{1} \geq \cdots \geq \sigma_{n}$. The equation (10) indicates that the matrix $P_{n \times m}$ can be expressed by $n$ characteristics. Each of the singular values and its eigenvectors represent a feature. If a certain singular value is set to be zero and the new components are reconstructed, the matrix $P_{n \times m}$ will lose the corresponding feature. It can realize the effect of filtering out unwanted signals.

\section{B. Proposed SOW-SVDR in Detail}

Firstly, the frequency information and the sampling rate of the measured signals should be confirmed. After arcing signal analysis under some loads, the authors have found that there is abundant arcing information in the frequency range of [0-400] $\mathrm{kHz}$. For example, the frequency-domain analysis on the arcing signals is shown in Fig. 8, when SAFs occur under the dimmer load. Therefore, according to the sampling theory, the sampling rate is selected as $1 \mathrm{MHz}$, and a low-pass filter (cut-off frequency: $400 \mathrm{kHz}$ ) is used to suppress the information higher than $400 \mathrm{kHz}$. The observation window will influence the time of identifying SAFs, which means it should be as short as possible. However, the arcing information will be insufficient for detection, if a too short observation window is used. Since the time-varying resistance of SAFs can be approximately 
regarded as a $0.01 \mathrm{~ms}$ periodic function, the arcing waveform can be also regarded as a periodic waveform. The observation window is selected as the integral multiples of the period to cover the periodic features. We have analyzed $0.01 \mathrm{~s}$ observation length, but it contains few arcing information. Therefore, the window length is selected as 0.02. Actually, according to UL1699, the observation length selection is not too strict, as long as the detection time is shorter than the required time. The observation window is selected as $0.02 \mathrm{~s}$. The number of samples $(u 1, u 2, u 3 \ldots u 20000)$ is $20000(=k)$ in every observation window. In the matrix $V$, the smaller of the two parameters, $n$ and $m$, influences the number of features. More features are helpful for correctly filtering out the non-arcing pulses. Thus, $n$ and $m$ are set as 100 and 200, respectively. The matrix $P$ is written below:

$$
P_{100 \times 200}=\left(\begin{array}{lll}
u_{1} & \ldots & u_{200} \\
\vdots & \ddots & \vdots \\
u_{19801} & \ldots & u_{20000}
\end{array}\right)
$$

After $\sigma_{1}$ and $\sigma_{2}$ is set as 0 , the changed components are reconstructed, which can solve the problem in Fig. 7. Fig. 9 shows the reconstructed waveforms from Fig. 7 (b), (c) and (d). The non-arcing pulses under the dimmer load are filtered out. In addition, most of the arcing ones are retained. Compared with other algorithms in Table II, the proposed method can enhance the identifying capability under the coupling method. In WT and reconstruction, the non-arcing pulses cannot be filtered out, because they exist in each decomposition layer by multi-resolution analysis. Then, correlation coefficient analysis, which is popular to evaluate waveform similarity for fault detection in power system, has been also tried to recognize the dimmer feature, cyclical fluctuation about $0.02 \mathrm{~s}$. In theory, the arcing coefficient should be small than the non-arcing one because of the low similarity among the arcing waveforms. However, the results are contrary to expectation, which indicates this algorithm has poor performance in practical experiments.

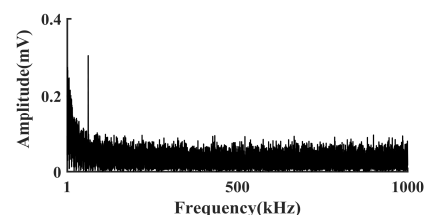

Fig. 8. Frequency analysis of coupling signals in the dimmer load.

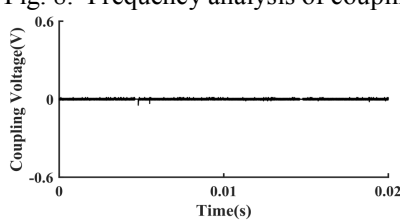

(a)

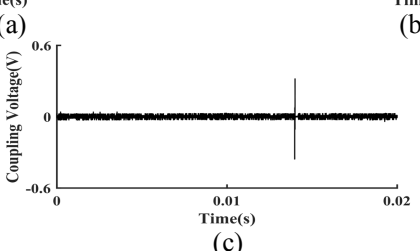

(c)

Fig. 9. Reconstructed waveforms from Fig. 7. (a) Normal coupling voltage waveform of the dimmer load. (b) Fault coupling voltage waveform of the resistance. (c) Fault coupling voltage waveform of the air compressor.
TABLE II

AbILity to Solve the Problem In Fig. 7 By Different Algorithms

\begin{tabular}{cc}
\hline \hline Method & $\begin{array}{c}\text { Ability to recognize the non-arcing } \\
\text { pulses }\end{array}$ \\
\hline Proposed SOW-SVDR & \multicolumn{1}{c}{$\begin{array}{c}\text { Strong } \\
\text { (can filter out the non-arcing pulses } \\
\text { while retaining the arcing ones) } \\
\text { Weak }\end{array}$} \\
$\begin{array}{c}\text { WT and reconstruction } \\
\text { (cannot filter out the non-arcing pulses } \\
\text { based on Pearson, Spearman } \\
\text { and Kendall, respectively }\end{array}$ & $\begin{array}{l}\text { Weak } \\
\text { (no difference between the arcing } \\
\text { coefficient and the normal one) }\end{array}$ \\
\hline \hline
\end{tabular}

The appliance starting process is a difficult problem for SAF detection. In traditional current detection, the starting current characteristics are similar to the arcing ones. There is also this problem in the coupling method. Many papers increase the number of detection cycles owing to the short starting time in order to avoid misjudgment caused by the staring process. This method increases additional time for identifying SAFs. Fortunately, SOW-SVDR can filter out the non-arcing pulses from the starting process and decrease the additional time used for it, which is proved in Fig. 10.

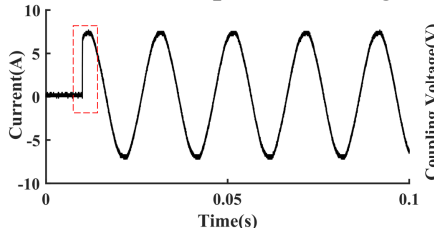

(a)

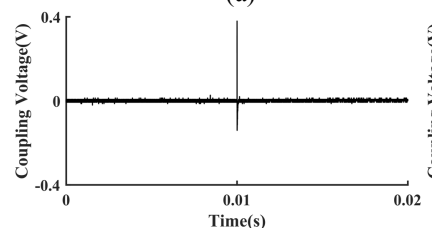

(c)

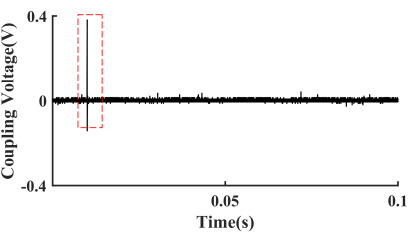

(b)

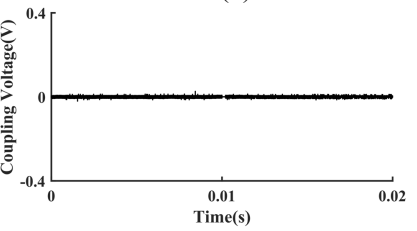

(d)
Fig. 10. Starting process in the resistance load. (a) Current waveform. (b) Coupling voltage waveform. (c) Coupling voltage waveform in a $20 \mathrm{~ms}$ observation window. (d) Reconstructed waveform of (c).

\section{SAF Detection Strategy}

The coupling method based on SOW-SVDR realizes satisfactory classification between arcing signals and non-arcing ones. When no SAF occurs, the coupling waveform is stable, and each sample amplitude is approximately zero. On the contrary, there are some pulses in the coupling waveform, when SAFs occur. According to this feature of the reconstructed signals, the second order cumulant algorithm (variance) is used to identify SAFs:

$$
E=\frac{1}{k} \sum_{i=1}^{k}\left(X_{i}-\mu\right)^{2}
$$

Where $k$ is the number of samples, and $\mu$ is the sample mean. The equation (12) is simplify as follows, because its division calculation plays a minor role here.

$$
E=\sum_{i=1}^{k}\left(X_{i}-\mu\right)^{2}
$$

The brief detection strategy is shown in Fig. 11. Finite difference that is sensitive to the arcing pulses is used for preprocessing the input data, which can enhance the ability to 
identify SAFs. Since it will lose one point, one more sample is required based on one observation window $(k=20000)$. Here, some loads are tested to verify the feasibility of the proposed strategy. The reason for selecting the $5 \mathrm{~A}$ resistor, the air compressor and the dimmer with three $200 \mathrm{~W}$ incandescent lamps (firing angle $90^{\circ}$ ) is that their waveforms under arcs and no arcs are very similar to each other, which provide a desirable testing scenario for validating the effectiveness of the proposed algorithm. Under each load, the arcing waveform and the non-arcing waveform are continuously calculated 10 times (200ms in total), respectively. The results are shown in Fig. 12 (a). The non-arcing variances range from 0.3 to 0.4 , and the arcing ones are above 1 . The starting process of the three loads is also tested, and their results are lower than 0.4 in Fig. 12 (b). Therefore, the threshold can be set as 0.6. If the acquired variance is smaller than 0.6 , it indicates there are no SAFs. Otherwise, it indicates there is a SAF. The experiments prove that variance recognizes the reconstructed signals well. Although the fourth order cumulant (kurtosis) also performs well in Table III, its computation is more complex than variance, because the computation complexity of $k$-th order cumulant increases with the increase of $k$.

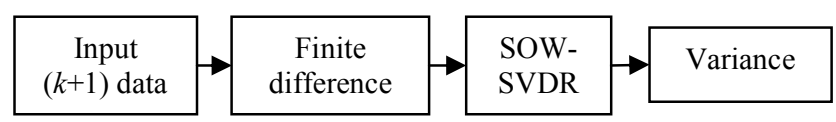

Fig. 11. Flowchart of the proposed strategy.

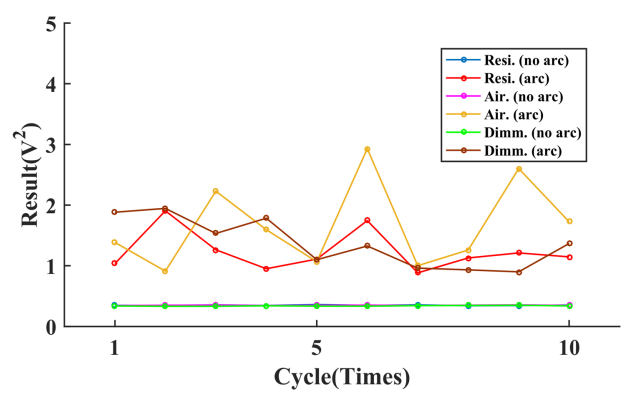

(a)

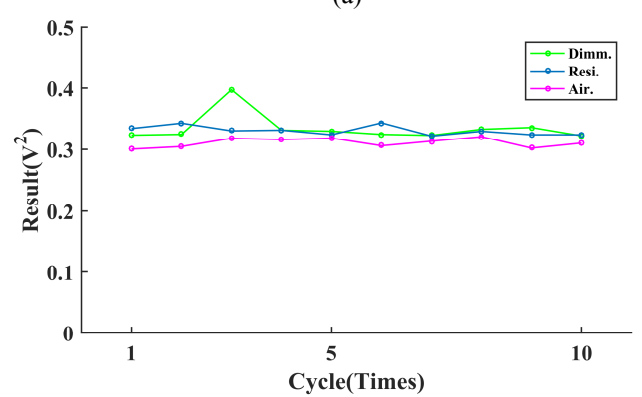

(b)

Fig. 12. Calculation results under arc and no arcs. (a) Operation state. (b) Starting process.

TABLE III

COMPARISON AMONG CUMULANT ALgORITHMS

\begin{tabular}{ccc}
\hline \hline Method & $\begin{array}{c}\text { Ability to recognize the } \\
\text { arcing pulses }\end{array}$ & $\begin{array}{c}\text { Computation } \\
\text { complexity }\end{array}$ \\
\hline $\begin{array}{c}\text { Second order cumulant } \\
\text { (variance) }\end{array}$ & Strong & $O(3 k)$ \\
Third order cumulant & Weak & $\mathrm{O}(4 \mathrm{k})$ \\
$\begin{array}{c}\text { Fourth order cumulant } \\
\text { (kurtosis) }\end{array}$ & Strong & $O(8 k)$ \\
\hline \hline
\end{tabular}

\section{EXPERIMENTAL VERIFICATIONS}

To further verify that whether the proposed strategy is appropriate for other kinds of loads and their combination, an experimental platform has been established according to UL1699, which is shown in Fig. 13 [35]. The arc generator can generate SAFs by separating the two rods in it. If SAFs are not needed, the two rods will be in contact with each other. The current transformer with a maximum bandwidth of $10 \mathrm{MHz}$ is chosen for acquire the high-frequency signals of SAFs. A second-order Butterworth low-pass filter is used to suppress high-frequency components above $400 \mathrm{kHZ}$. For correct data acquisition, the oscilloscope should be isolated from arc signals and connected to a $220 \mathrm{~V}$ isolated voltage source. LOAD 1 and LOAD 2 include the 5A resistance, the dimmer with $600 \mathrm{~W}$ incandescent lamps (firing angle $60^{\circ}, 90^{\circ}$ and $120^{\circ}$, respectively), the air compressor, the vacuum cleaner, the air compressor, the personal computer and the fluorescent lamps. In the single load experiments, the switch $\mathrm{S} 1$ is turned on, and the switch S2 is turned off to realize the trunk fault. Then, each of the above-mentioned loads is tested. In the combined load experiments, both of the two switches S1 and S2 are turned on to realize the branch fault, and one of the branch loads is selected as the dimmer with $600 \mathrm{~W}$ lamps, because the circuits involving the dimmer load often lead to unwanted trips. Additionally, the $200 \mathrm{~W}$ lamp is tested in parallel with the $10 \mathrm{~A}$ resistance, where the failure-to-trip situations often occur. The test results are from the normal condition, the fault condition and the starting condition, respectively, and recorded in Table IV, where L1 and L2 mean that the corresponding loads are put in LOAD 1 and LOAD 2 of Fig. 13, respectively. For more analytical description of the experimental procedure, some typical examples in Table IV are taken to introduce how to do the experiments. "L1: Resistance (5A)" means that the 5A resistance is put in LOAD 1 after the switch $\mathrm{S} 1$ is turned on (the switch S2 keeps disconnected). "L1: res. 5A+L2: Dim. 600W lamps $\left(60^{\circ}\right)$ " means that the resistance and the dimmer are put in LOAD 1 and LOAD 2, respectively, after the switches S1 and $\mathrm{S} 2$ are turned on. When the loads are working in the normal and arcing conditions, the acquired waveforms are calculated to obtain the corresponding variance results. After the loads are turned on, the starting waveforms are captured and calculated for obtaining the variance results during the starting process. All of the normal results and the starting ones are in [0.3-0.4], and the fault results are much bigger than 0.4 .

In Table $\mathrm{V}$, the proposed strategy is compared with some other strategies published recently. Reference [32] utilizes kurtosis and counts the number of pulses to identify arcing signals. The proposed strategy appears to fail to work in case with the dimmer load, because either the first normal indicator $(N I)$ or the second normal one will sometimes be very large, which is bigger than the threshold and misjudges there is a SAF. Reference [33] considers the non-arcing pulses distribute rarely, and the arcing ones distribute abundantly. It divides a long observation window into many small ones according to the time width of the normal pulses in the dimmer load. However, the time width of the normal pulses is changing, which sometimes makes the fault indicators $(F I)$ smaller than the thresholds to cause missed judgment and probably results in detection instability. In appliance starting process, these references 
cannot solve the unwanted judgment and have to increase many detection cycles to avoid it, which will delay much time for detecting SAFs.

TABLE IV

CALCULATION RESUltS IN DifFERENT LOADS

\begin{tabular}{|c|c|c|c|c|}
\hline Load & $\begin{array}{l}\text { Number } \\
\text { of cycles } \\
\text { (times) }\end{array}$ & $\begin{array}{c}\text { Enormal } \\
\qquad\left(\mathrm{V}^{2}\right)\end{array}$ & $\begin{array}{l}\text { Earcing } \\
\qquad\left(\mathrm{V}^{2}\right)\end{array}$ & $\begin{array}{c}\text { Estartig- } \\
\text { process } \\
\left(\mathrm{V}^{2}\right)\end{array}$ \\
\hline L1: Resistance (5A) & 200 & $<0.35$ & $>0.82$ & $<0.35$ \\
\hline $\begin{array}{l}\text { L1: Dimmer with } \\
600 \mathrm{~W} \text { lamps }\left(60^{\circ}\right)\end{array}$ & 100 & $<0.37$ & $>1.01$ & $<0.38$ \\
\hline $\begin{array}{l}\text { L1: Dimmer with } \\
600 \mathrm{~W} \text { lamps }\left(90^{\circ}\right)\end{array}$ & 100 & $<0.36$ & $>0.91$ & $<0.39$ \\
\hline $\begin{array}{l}\text { L1: Dimmer with } \\
600 \mathrm{~W} \text { lamps }\left(120^{\circ}\right)\end{array}$ & 100 & $<0.36$ & $>1.11$ & $<0.37$ \\
\hline L1: Personal computer & 100 & $<0.32$ & $>4.21$ & $<0.39$ \\
\hline L1: Air compressor & 200 & $<0.35$ & $>0.85$ & $<0.33$ \\
\hline L1: Vacuum cleaner & 200 & $<0.34$ & $>1.83$ & $<0.35$ \\
\hline $\begin{array}{l}\text { L1: Fluorescent lamps } \\
(80 \mathrm{~W})+5 \mathrm{~A} \text { resistance }\end{array}$ & 100 & $<0.39$ & $>1.2$ & $<0.34$ \\
\hline $\begin{array}{l}\mathrm{L} 1: \text { res. } 5 \mathrm{~A}+\mathrm{Dim} . \\
600 \mathrm{~W} \text { lamps }\left(60^{\circ}\right)\end{array}$ & 100 & $<0.36$ & $>0.92$ & $<.0 .36$ \\
\hline $\begin{array}{l}\mathrm{L} 1: \text { res. } 5 \mathrm{~A}+\mathrm{L} 2: \mathrm{Dim} . \\
600 \mathrm{~W} \text { lamps }\left(60^{\circ}\right)\end{array}$ & 100 & $<0.35$ & $>0.79$ & $<0.36$ \\
\hline $\begin{array}{l}\text { L1: Dim. 600W lamps } \\
\left(60^{\circ}\right)+\mathrm{L} 2 \text { : res. } 5 \mathrm{~A}\end{array}$ & 100 & $<0.35$ & $>1.08$ & $<0.36$ \\
\hline $\begin{array}{l}\mathrm{L} 1: 200 \mathrm{~W} \text { lamp+L2: } \\
\text { res. } 10 \mathrm{~A}\end{array}$ & 200 & $<0.32$ & $>1.0$ & $<0.34$ \\
\hline
\end{tabular}

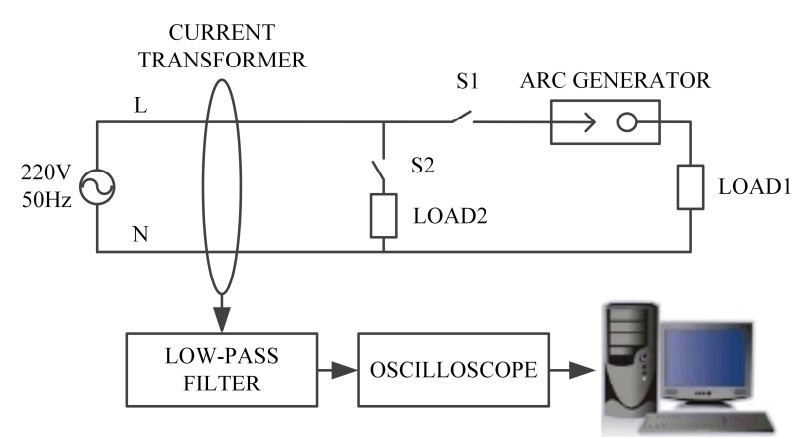

(a)

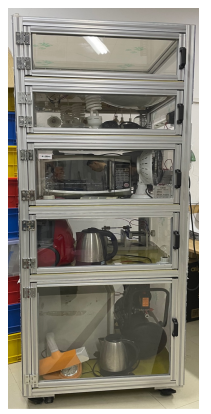

(b)

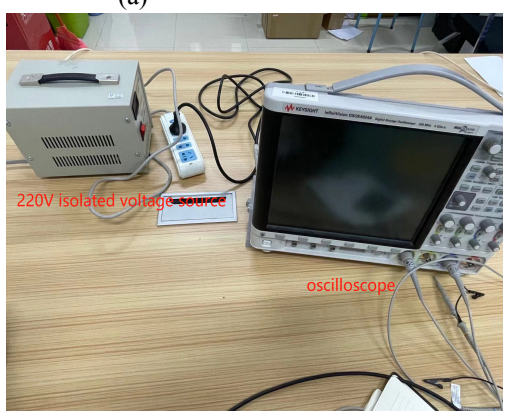

(c)
Fig. 13. Experimental introduction. (a) Schematic of experimental platform. (b) Fault-generation setup. (c) Data acquisition setup.
TABLE V

Comparison of the Performance of Different Detection Strategies

\begin{tabular}{|c|c|c|c|c|}
\hline $\begin{array}{l}\text { Detection } \\
\text { strategy }\end{array}$ & $\begin{array}{l}\text { Sampling } \\
\text { rate }\end{array}$ & $\begin{array}{l}\text { Circuits with } \\
\text { the dimmer }\end{array}$ & $\begin{array}{l}\text { Circuits with } \\
\text { other loads }\end{array}$ & $\begin{array}{l}\text { Starting } \\
\text { process }\end{array}$ \\
\hline [32] & $10 \mathrm{MHz}$ & $\begin{array}{l}\text { Fail to work } \\
\text { ( } N I \geq \text { threshold } \\
F I \geq \text { threshold) }\end{array}$ & $\begin{array}{l}\text { Work well } \\
(N I<\text { threshold } \\
F I \geq \text { threshold })\end{array}$ & $\begin{array}{l}\text { Fail to work } \\
(N I \geqslant \text { threshold })\end{array}$ \\
\hline [33] & $1 \mathrm{MHz}$ & $\begin{array}{l}\text { Not reliable } \\
(N I<\text { threshold } \\
F I<\text { threshold })\end{array}$ & $\begin{array}{l}\text { Work well } \\
(N I<\text { threshold } \\
F I \geq \text { threshold })\end{array}$ & $\begin{array}{l}\text { Fail to work } \\
(N I \geqslant \text { threshold })\end{array}$ \\
\hline $\begin{array}{l}\text { Proposed } \\
\text { strategy }\end{array}$ & $1 \mathrm{MHz}$ & $\begin{array}{l}\text { Work well } \\
(N I<\text { threshold } \\
F I \geq \text { threshold })\end{array}$ & $\begin{array}{l}\text { Work well } \\
(N I<\text { threshold } \\
F I \geq \text { threshold })\end{array}$ & $\begin{array}{l}\text { Work well } \\
(N I<\text { threshold })\end{array}$ \\
\hline
\end{tabular}

\section{CONCLUSION}

In this paper, a coupling method based on SOW-SVDR is proposed to solve misjudgment and missed judgment caused by the similarity between arcing and non-arcing signals. The following key conclusions and findings were made from this work:

(1) The traditional current detection methods, which are based on FFT, WT and CZT, are not capable of extracting the small-amplitude fault features from the trunk-line current, even if high frequency resolution is adopted. Compared with these methods, the proposed coupling method, which acquires differential magnetic flux in the iron core, can effectively solve the problem associated with the small-amplitude fault features in the trunk-line current. It is convenient for practical application to pass the neutral line and the live line through the current sensor without any additional hardware design. When most loads are working under normal conditions, the corresponding output shows flat waveform with approximately zero output. When they are working in the fault condition, the output shows a series of clear pulses.

(2) Similar to the traditional method, the proposed coupling method experience mal-detection issues in the dimmer load and appliance starting process, where the normal signals also have fault-like features. Furthermore, it is difficult to filter out these undesirable features by some popular algorithms, such as WT and the like, because their frequency bands are wide. To solve this problem, the SOW-SVDR algorithm, which can decompose signals into many components and reconstruct them in a short observation window, is proposed to filter out the unwanted signals and retain the useful ones for fast detecting SAFs. The coupling method based on SOW-SVDR can realize the signal classification effect in SAF detection, which is suitable to recognize SAFs by simple algorithms.

(3) According to the signal characteristics processed by the coupling method based on SOW-SVDR, the second order cumulant algorithm (variance), which is simpler than high order cumulant in computation complexity, is used to judge whether there is a SAF. The experimental verification has demonstrated that the proposed detection strategy can be used for effective detection and identification of SAFs.

\section{REFERENCES}

[1] Q. Xiong et al., "Series Arc Fault Detection and Localization in DC Distribution System," IEEE Transactions on Instrumentation and Measurement, vol. 69, no. 1, pp. 122-134, Jan. 2020. 
[2] W. Moon, J. Kim, A. Jo, S. Bang and W. Koh, "Ignition Characteristics of Residential Series Arc Faults in 220-V HIV Wires," IEEE Transactions on Industry Applications, vol. 51, no. 3, pp. 2054-2059, May-June 2015.

[3] L. Zhao, Y. Zhou, K. Chen, S. Rau and W. Lee, "High-Speed Arcing Fault Detection: Using the Light Spectrum," IEEE Industry Applications Magazine, vol. 26, no. 3, pp. 29-36, May-June 2020.

[4] J. C. Kim, D. O. Neacşu, R. Ball and B. Lehman, "Clearing Series AC Arc Faults and Avoiding False Alarms Using Only Voltage Waveforms," IEEE Transactions on Power Delivery, vol. 35, no. 2, pp. 946-956, April 2020.

[5] Q. Lu, Z. Ye, Y. Zhang, T. Wang, Z. Gao, "Analysis of the Effects of Arc Volt-ampere Characteristics on Different Loads and Detection Methods of Series Arc Faults, Energies," vol. 12, no. 2, Jan. 2019.

[6] P. Parikh, D. Allcock, R. Luna and J. Vico, "A Novel Approach for Arc-Flash Detection and Mitigation: At the Speed of Light and Sound," IEEE Transactions on Industry Applications, vol. 50, no. 2, pp. 1496-1502, March-April 2014.

[7] G. Wang, W. Kim, H. Ji, G. Kil, "Detection and Analysis of series arc using non-conventional methods in low-voltage switchboards," Journal of electrical engineering-elektrotechnicky casopis, vol. 69, no. 4, pp. 317-322, Aug. 2018.

[8] R. M. Harris, M. D. Judd, P. J. Moore and J. Livie, "Radiometric detection and analysis of arcing faults," IEEE Transactions on Dielectrics and Electrical Insulation, vol. 22, no. 3, pp. 1547-1558, June 2015.

[9] J. P. Pulkkinen, "Commercial arc fault detection devices in military electromagnetic environment," IEEE Electromagnetic Compatibility Magazine, vol. 7, no. 4, pp. 49-52, 4th Quarter 2018.

[10] A. Mukherjee, A. Routray and A. K. Samanta, "Method for Online Detection of Arcing in Low-Voltage Distribution Systems," IEEE Transactions on Power Delivery, vol. 32, no. 3, pp. 1244-1252, June 2017.

[11] S. A. Saleh, M. E. Valdes, C. S. Mardegan and B. Alsayid, "The State-of-the-Art Methods for Digital Detection and Identification of Arcing Current Faults," in IEEE Transactions on Industry Applications, vol. 55, no. 5, pp. 4536-4550, Sept.-Oct. 2019.

[12] E. Calderon, P. Schweitzer, S. Weber. "Kalman Filter and a Fuzzy Logic Processor for Series Arcing Fault Detection in a Home Electrical Network," International Journal of Electrical Power \& Energy Systems, vol. 107, pp. 251-263, May. 2019.

[13] Etienne Tisserand, Jinmi Lezama, Patrick Schweitzer, Yves Berviller, "Series arcing detection by algebraic derivative of the current," Electric Power Systems Research, vol. 119, pp. 91-99, Feb. 2015.

[14] C. J. Kim, "Electromagnetic Radiation Behavior of Low-Voltage Arcing Fault," IEEE Transactions on Power Delivery, vol. 24, no. 1, pp. 416-423, Jan. 2009.

[15] M. K. Khafidli, E. Prasetyono, D. O. Anggriawan, A. Tjahjono and M. H. Riza Alvi Syafii, "Implementation AC Series Arc Fault Recognition using Mikrokontroller Based on Fast Fourier Transform," 2018 International Electronics Symposium on Engineering Technology and Applications (IES-ETA), Bali, 2018, pp. 31-36.

[16] G. Artale, A. Cataliotti, V. Cosentino, D. Di Cara, S. Nuccio and G. Tinè, "Arc Fault Detection Method Based on CZT Low-Frequency Harmonic Current Analysis," in IEEE Transactions on Instrumentation and Measurement, vol. 66, no. 5, pp. 888-896, May 2017.

[17] N. Qu, J. Wang and J. Liu, "An Arc Fault Detection Method Based on Current Amplitude Spectrum and Sparse Representation," in IEEE Transactions on Instrumentation and Measurement, vol. 68, no. 10, pp. 3785-3792, Oct. 2019.

[18] E. Karakose, MT. Gencoglu, M. Karakose, O. Yaman, I. Aydin, E. Akin, "A new arc detection method based on fuzzy logic using S-transform for pantograph-catenary systems," Journal of Intelligent Manufacturing, vol. 29, no. 4, pp. 839-856, Apr. 2018.

[19] X. Qin, P. Wang, Y. Liu, L. Guo, G. Sheng and X. Jiang, "Research on Distribution Network Fault Recognition Method Based on Time-Frequency Characteristics of Fault Waveforms," IEEE Access, vol. 6, pp. 7291-7300, August. 2018.

[20] A. F. Ilman and Dzulkiflih, "Low Voltage Series Arc Fault Detecting With Discrete Wavelet Transform," 2018 International Conference on Applied Engineering (ICAE), Batam, October. 2018, pp. 1-5.

[21] J. Hong-Keum, C. Young-Jin, W. GuoMing, H. Seong-Cheol and K. Gyung-Suk, "Extraction of Series Arc Signals Based on Wavelet Transform in an Indoor Wiring System," Transactions on Electrical and Electronic Materials, vol. 18, no.4, pp. 221-224, August. 2017.
[22] H. Ji, G. Wang, W. Kim, G. Kil, "Optimal Design of A Band Pass Filter and an Algorithm for Series Arc Fault Detection," Energies, vol. 11, no. 4, Apr. 2018.

[23] M. Wei et al., "High Impedance Arc Fault Detection Based on the Harmonic Randomness and Waveform Distortion in the Distribution System," IEEE Transactions on Power Delivery, vol. 35, no. 2, pp. 837-850, April 2020.

[24] F. Guo, H. Gao, Z. Wang, J. You, A. Tang and Y. Zhang, "Detection and Line Selection of Series Arc Fault in Multi-Load Circuit," IEEE Transactions on Plasma Science, vol. 47, no. 11, pp. 5089-5098, Nov. 2019.

[25] Y. Wang, F. Zhang, X. Zhang and S. Zhang, "Series AC Arc Fault Detection Method Based on Hybrid Time and Frequency Analysis and Fully Connected Neural Network," IEEE Transactions on Industrial Informatics, vol. 15, no. 12, pp. 6210-6219, Dec. 2019.

[26] Y. Wang, F. Zhang and S. Zhang, "A New Methodology for Identifying Arc Fault by Sparse Representation and Neural Network," IEEE Transactions on Instrumentation and Measurement, vol. 67, no. 11, pp. 2526-2537, Nov. 2018.

[27] J. Jiang et al., "Series Arc Detection and Complex Load Recognition Based on Principal Component Analysis and Support Vector Machine," IEEE Access, vol. 7, pp. 47221-47229, 2019.

[28] J. C. Kim, D. O. Neacşu, R. Ball and B. Lehman, "Clearing Series AC Arc Faults and Avoiding False Alarms Using Only Voltage Waveforms," IEEE Transactions on Power Delivery, vol. 35, no. 2, pp. 946-956, April 2020.

[29] N. Qu, J. Zuo, J. Chen and Z. Li, "Series Arc Fault Detection of Indoor Power Distribution System Based on LVQ-NN and PSO-SVM," IEEE Access, vol. 7, pp. 184020-184028, 2019.

[30] Y. Kai, C. Ruobo, Z. Rencheng, X. Jinchao and T. Ran, "A Novel Methodology for Series Arc Fault Detection by Temporal Domain Visualization and Convolutional Neural Network, "Sensors, vol. 20, no. 1, Dec, 2019.

[31] K. Li, S. Zhao and Y. Wang, "A Planar Location Method for DC Arc Faults Using Dual Radiation Detection Points and DANN," IEEE Transactions on Instrumentation and Measurement, vol. 69, no. 8, pp. 5478-5487, Aug. 2020.

[32] G. Bao and R. Jiang, "Research on Series Arc Fault Detection Based on Asymmetrical Distribution of Magnetic Flux, " Chinese Journal of Scientific Instrument, vol. 40, no. 3, pp. 54-61, Mar, 2019.

[33] G. Bao, R. Jiang and X. Gao, "Novel Series Arc Fault Detector Using High-Frequency Coupling Analysis and Multi-Indicator Algorithm," IEEE Access, vol. 7, pp. 92161-92170, 2019.

[34] J. Zhong, X. Bi, Q. Shu, M. Chen, D. Zhou and D. Zhang, "Partial Discharge Signal Denoising Based on Singular Value Decomposition and Empirical Wavelet Transform," IEEE Transactions on Instrumentation and Measurement, vol. 69, no. 11, pp. 8866-8873, Nov. 2020.

[35] Standard for Arc-Fault Circuit Interrupters, UL Standard 1699, Apr. 2006. 